\title{
Generation of Alkoxycarbenium Ion Pools from Thioacetals and Applications to Glycosylation Chemistry
}

Shinkiti Suzuki, Kouichi Matsumoto, Kohsuke Kawamura, Seiji Suga, and Jun-ichi Yoshida* Department of Synthetic Chemistry and Biological Chemistry, Graduate School of Engineering, Kyoto University, Nishikyo-ku, Kyoto 615-8510, Japan.

Materials. Dichloromethane was washed with water, distilled from $\mathrm{P}_{2} \mathrm{O}_{5}$, redistilled from dried $\mathrm{K}_{2} \mathrm{CO}_{3}$ to remove a trace amount of acid, and stored over molecular sieves $4 \mathrm{~A}$. $\alpha$-Phenylthio ethers $1,{ }^{1} 5,{ }^{2}$ and $\mathbf{6}^{2}$ were prepared according to the procedures in the literatures. Thioglycosides $\mathbf{9}^{3,14,15}$ and $\mathbf{1 0}^{14,16}$ were prepared according to the literature. Glycosyl acceptors $\mathbf{1 9}^{3}, \mathbf{2 2}^{4}$ were prepared as reported. Compounds $4,{ }^{13} 7,{ }^{13}$ and $\mathbf{8},{ }^{13}$ glycosides $11,{ }^{7} \mathbf{1 2},{ }^{5} \mathbf{1 3},{ }^{10} \mathbf{1 4},{ }^{10} \mathbf{1 5},{ }^{8} \mathbf{1 6},{ }^{6} \mathbf{1 7},{ }^{9} \mathbf{1 8},{ }^{6}$ and $\mathbf{2 0}^{9,11}$ obtained by the present method were characterized by comparison of their ${ }^{1} \mathrm{H}$ NMR spectra with those of authentic samples reported in the literature.

Generation of Alkoxycarbenium Ions. Typical Procedure. The anodic oxidation was carried out in an H-type divided cell (4G glass filter) equipped with a carbon felt anode (Nippon Carbon JF-20-P7, ca. $320 \mathrm{mg}$, dried at $250{ }^{\circ} \mathrm{C} / 1 \mathrm{mmHg}$ for $1 \mathrm{~h}$ before use) and a platinum plate cathode $(40 \mathrm{~mm} \times 20 \mathrm{~mm})$. In the anodic chamber was placed a solution of $\alpha$-phenylthioether $(0.4$ mmol) in $0.3 \mathrm{M} \mathrm{Bu}_{4} \mathrm{NBF}_{4} / \mathrm{CH}_{2} \mathrm{Cl}_{2}(8.0 \mathrm{~mL})$. In the cathodic chamber were placed $0.3 \mathrm{M}$ $\mathrm{Bu}_{4} \mathrm{NBF}_{4} / \mathrm{CH}_{2} \mathrm{Cl}_{2}(8.0 \mathrm{~mL})$ and trifluoromethanesulfonic acid $(150.1 \mathrm{mg}, 0.4 \mathrm{mmol})$. The constant current electrolysis $(8 \mathrm{~mA})$ was carried out at $-78{ }^{\circ} \mathrm{C}$ with magnetic stirring until $2.5 \mathrm{~F} / \mathrm{mol}$ of electricity was consumed.

NMR Analysis of Alkoxycarbenium Ion (2). ${ }^{1} \mathrm{H}$ and ${ }^{13} \mathrm{C}$ NMR spectra were recorded in $\mathrm{CD}_{2} \mathrm{Cl}_{2}$ on a JEOL A-500 spectrometer. Chemical shifts are reported using methylene signals at $\delta$ $5.32\left({ }^{1} \mathrm{H}\right.$ NMR $)$ and $\delta 53.80\left({ }^{13} \mathrm{C}\right.$ NMR $)$ as standards. The anodic oxidation was carried out in a divided cell equipped with a carbon felt anode and a platinum plate cathode. In the anodic chamber were placed a solution of 1-methoxy-1-phenylthiononane (1) $(40.3 \mathrm{mg}, 0.175 \mathrm{mmol})$ in $3.5 \mathrm{~mL}$ of $0.3 \mathrm{M} \mathrm{Bu}_{4} \mathrm{NBF}_{4} / \mathrm{CD}_{2} \mathrm{Cl}_{2}$. In the cathodic chamber were placed trifluoromethanesulfonic acid (29.7 $\mathrm{mg}, 0.198 \mathrm{mmol})$ and $0.3 \mathrm{M} \mathrm{Bu}_{4} \mathrm{NBF}_{4} / \mathrm{CD}_{2} \mathrm{Cl}_{2}(3.5 \mathrm{~mL})$. The constant current electrolysis $(3.5 \mathrm{~mA})$ was carried out at $-78{ }^{\circ} \mathrm{C}$ with magnetic stirring. After $1.4 \mathrm{~F} / \mathrm{mol}$ of electricity was consumed, the reaction mixture of the anodic chamber was transferred to a $5 \mathrm{~mm} \phi$ NMR tube with a septum cap under Ar atmosphere at $-78{ }^{\circ} \mathrm{C}$. The NMR measurement was carried out at $-80{ }^{\circ} \mathrm{C}:{ }^{1} \mathrm{H}$ NMR $(500$ $\mathrm{MHz}, \mathrm{CD}_{2} \mathrm{Cl}_{2}$, selected) $\delta 4.95(\mathrm{~s}, 3 \mathrm{H}), 9.55(\mathrm{~s}, 1 \mathrm{H}) ;{ }^{13} \mathrm{C} \mathrm{NMR}\left(125.65 \mathrm{MHz}, \mathrm{CD}_{2} \mathrm{Cl}_{2}\right.$, selected) $\delta$ $40.7\left(\mathrm{CH}_{3} \mathrm{OCHCH}_{2}\right), 76.1\left(\underline{\mathrm{CH}}_{3} \mathrm{OCH}\right), 230.9\left(\mathrm{CH}_{3} \mathrm{O} \underline{\mathrm{CH}}\right)$. In the NMR spectra the other signals could not be assigned because of overlap of the signals of $\mathrm{Bu}_{4} \mathrm{NBF}_{4}$ used as electrolyte.

The signals due to the thiophenyl group leaving from 1 were also observed: ${ }^{1} \mathrm{H}$ NMR $\delta$ 7.2-8.0 (m); ${ }^{13} \mathrm{C}$ NMR $\delta 116.5,119.0,124.1,130.3$.

4-Methoxy-dodec-1-ene (4). A Typical Procedure of Reaction between Alkoxycarbenium Ion and Carbon Nucleophile. The electrolysis of $\mathbf{1}$ (91.6 mg, $0.397 \mathrm{mmol}$ ) was carried out as described above. To the "cation pool" thus generated in the anodic chamber, was added allyltrimethylsilane $\left(93.9 \mathrm{mg}, 0.822 \mathrm{mmol}\right.$ ) at $-78{ }^{\circ} \mathrm{C}$ and the reaction mixture was stirred for $10 \mathrm{~min}$. The solvent was removed under reduced pressure and the residue was quickly filtered 
through a short column $(2 \times 3 \mathrm{~cm})$ of silica gel to remove $\mathrm{Bu}_{4} \mathrm{NBF}_{4}$. The silica gel was washed with ether $(150 \mathrm{~mL})$. The GC analysis of the combined filtrate indicated that $\mathbf{4}$ was formed in $80 \%$ yield (GC ${ }^{t} R 6.3 \mathrm{~min}$, column, OV-1; $0.25 \mathrm{~mm} \times 25 \mathrm{~m}$; initial oven temperature, $100{ }^{\circ} \mathrm{C}$; rate of temperature increase, $10{ }^{\circ} \mathrm{C} / \mathrm{min}$ ): TLC $R_{f} 0.38$ (hexane/EtOAc 10:1); ${ }^{1} \mathrm{H}$ NMR $\left(300 \mathrm{MHz}, \mathrm{CDCl}_{3}\right.$ ) $\delta 0.88(\mathrm{t}, J=6.6 \mathrm{~Hz}, 3 \mathrm{H}), 1.16-1.40(\mathrm{~m}, 12 \mathrm{H}), 1.40-1.53(\mathrm{~m}, 2 \mathrm{H}), 2.24-2.28(\mathrm{~m}, 2 \mathrm{H}), 3.16-3.24(\mathrm{~m}$, $2 \mathrm{H}), 3.34(\mathrm{~s}, 3 \mathrm{H}), 5.03-5.19(\mathrm{~m}, 2 \mathrm{H}), 5.75-5.89(\mathrm{~m}, 1 \mathrm{H}) ;{ }^{13} \mathrm{C} \mathrm{NMR}\left(75 \mathrm{MHz}, \mathrm{CDCl}_{3}\right) \delta 14.0,22.6$, 25.2, 29.2, 29.5, 29.7, 31.8, 33.3, 37.7, 56.5, 80.5, 116.7, 135.1; IR (neat) 2928, 1100, $912 \mathrm{~cm}^{-1}$; LRMS (EI) m/e $197\left(\mathrm{M}^{+}-\mathrm{H}\right), 157\left(\mathrm{M}^{+}-\mathrm{CH}_{2} \mathrm{CH}=\mathrm{CH}_{2}\right)$; HRMS (EI) calcd for $\mathrm{C}_{13} \mathrm{H}_{25} \mathrm{O}\left(\mathrm{M}^{+}-\mathrm{H}\right)$ : 197.1905, found: 197.1915; Anal. Calcd for $\mathrm{C}_{13} \mathrm{H}_{26} \mathrm{O}$ : C, 78.72; H, 13.21. Found: C, 78.79; H, 13.13.

Glycosylation by Low-Temperature Electrolysis in the Absence of Glycosyl Acceptors (One-Pot Procedure). The anodic oxidation was carried out in an $\mathrm{H}$-type divided cell (4G glass filter) equipped with a carbon felt anode (Nippon Carbon JF-20-P7, ca. $320 \mathrm{mg}$, dried at $250{ }^{\circ} \mathrm{C} / 1$ $\mathrm{mmHg}$ for $1 \mathrm{~h}$ before use) and a platinum plate cathode (40 mm x $20 \mathrm{~mm})$. In the anodic chamber was placed a solution of $\mathbf{1 0}(65.7 \mathrm{mg}, 0.2 \mathrm{mmol})$ in $0.1 \mathrm{M} \mathrm{Bu}_{4} \mathrm{NClO}_{4} / \mathrm{CH}_{2} \mathrm{Cl}_{2}(12.0 \mathrm{~mL})$. In the cathodic chamber were placed $0.1 \mathrm{M} \mathrm{Bu}_{4} \mathrm{NClO}_{4} / \mathrm{CH}_{2} \mathrm{Cl}_{2}(12.0 \mathrm{~mL})$ and trifluoromethanesulfonic acid $(150.1 \mathrm{mg}, 0.4 \mathrm{mmol})$. The constant current electrolysis $(4 \mathrm{~mA})$ was carried out at $-78{ }^{\circ} \mathrm{C}$ with magnetic stirring until $1.25 \mathrm{~F} / \mathrm{mol}$ of electricity was consumed. To the solution thus obtained in the anodic chamber, was added methanol $(32.0 \mathrm{mg}, 1.0 \mathrm{mmol})$. The reaction mixture was stirred for 1-2 min at $-78{ }^{\circ} \mathrm{C}$. After triethylamine $(40.5 \mathrm{mg}, 0.4 \mathrm{mmol})$ was added, the reaction mixture was warmed to room temperature. The solvent was removed under reduced pressure and the residue was quickly filtered through a short column $(2 \times 10 \mathrm{~cm})$ of silica gel to remove $\mathrm{Bu}_{4} \mathrm{NClO}_{4}$. The silica gel was washed with ether $(150 \mathrm{~mL})$. The combined filtrate was purified with flash chromatography (hexane/EtOAc $3: 1$ to $1: 1$ ) to give $\mathbf{1 2} \alpha$ and $\mathbf{1 2} \beta$ (total $33.7 \mathrm{mg}, 67 \%$, diastereomer ratio was $\alpha / \beta=$ $15: 85)$.

Glycosylation by Low-Temperature Electrolysis in the Presence of Glycosyl Acceptors: (In-Situ Procedure). The anodic oxidation was carried out in an H-type divided cell (4G glass filter) equipped with a carbon felt anode (Nippon Carbon JF-20-P7, ca. $320 \mathrm{mg}$, dried at $250{ }^{\circ} \mathrm{C} / 1 \mathrm{mmHg}$ for $1 \mathrm{~h}$ before use) and a platinum plate cathode (40 mm x $20 \mathrm{~mm}$ ). In the anodic chamber was placed a solution of $10(65.9 \mathrm{mg}, 0.201 \mathrm{mmol})$ and methanol $(63.8 \mathrm{mg}, 1.991 \mathrm{mmol})$ in $0.1 \mathrm{M} \mathrm{Bu} \mathrm{NTEPB} / \mathrm{CH}_{2} \mathrm{Cl}_{2}(12.0 \mathrm{~mL})$. In the cathodic chamber were placed $0.1 \mathrm{M}$ $\mathrm{Bu}_{4} \mathrm{TEPB} / \mathrm{CH}_{2} \mathrm{Cl}_{2}(12.0 \mathrm{~mL})$ and trifluoromethanesulfonic acid $(80.8 \mathrm{mg}, 0.538 \mathrm{mmol})$. The constant current electrolysis $(4 \mathrm{~mA})$ was carried out at $-78{ }^{\circ} \mathrm{C}$ with magnetic stirring until 1.25 $\mathrm{F} / \mathrm{mol}$ of electricity was consumed. After addition of triethylamine $(48.2 \mathrm{mg}, 0.476 \mathrm{mmol})$, the reaction mixture was warmed to room temperature. The solvent was removed under reduced pressure and the residue was dissolved in EtOAc $(1 \mathrm{~mL})$, and then hexane $(10 \mathrm{~mL})$ was added. $\mathrm{Bu}_{4}$ TEPB precipitated. After the solution phase was separated by decantation, the solid materials were dissolved in EtOAc $(1 \mathrm{~mL})$ and hexane $(10 \mathrm{~mL})$ was added. $\mathrm{Bu}_{4}$ TEPB precipitated. After the solution phase was separated by decantation, the solid materials were dissolved in EtOAc $(1 \mathrm{~mL})$ and hexane $(10 \mathrm{~mL})$ was added. $\mathrm{Bu}_{4}$ TEPB precipitated (three times in total). The solution phase was separated by decantation. The solution phase was combined and the solvent was removed with a rotary evaporator under reduced pressure to give the crude product. Purified with flash chromatography (hexane/EtOAc 3:1 to $1: 1$ ) to give $\mathbf{1 2} \alpha$ and $\mathbf{1 2} \beta$ (total $45.3 \mathrm{mg}, 90 \%$, diastereomer ratio was $\alpha / \beta=63: 37)$. 
Methyl-6-O-(2,3,4,6-tetra- $O$-methyl- $\alpha$-D-glucopyranosyl)-2,3,4-tri- $O$-benzyl- $\alpha$-D-gluco pyranoside $(\mathbf{2 1 \alpha}){ }^{12}$ Prepared from $10(65.7 \mathrm{mg}, 0.200 \mathrm{mmol})$ and 19 (914.8 $\left.\mathrm{mg}, 1.969 \mathrm{mmol}\right)$ in the in-situ procedure, and purified with flash chromatography (hexane/EtOAc 5:3 to 1:1) (111.3 mg, $82 \%, \alpha: \beta=67: 33$ ). TLC $R_{f} 0.34$ (hexane/EtOAc $\left.1: 1\right) ;{ }^{1} \mathrm{H}$ NMR $\left(400 \mathrm{MHz}, \mathrm{CDCl}_{3}\right) \delta 3.16(\mathrm{dd}, J=$ 3.6, $9.6 \mathrm{~Hz}, 1 \mathrm{H}), 3.19$ (dd, $J=8.8,10.0 \mathrm{~Hz}, 1 \mathrm{H}), 3.37$ (s, 3H), 3.38 (s, 3H), 3.38 (s, 3H), 3.44-3.66 $(\mathrm{m}, 6 \mathrm{H}), 3.52(\mathrm{~s}, 3 \mathrm{H}), 3.61(\mathrm{~s}, 3 \mathrm{H}), 3.72-3.81(\mathrm{~m}, 3 \mathrm{H}), 4.00(\mathrm{dd}, J=9.2,9.2 \mathrm{~Hz}, 1 \mathrm{H}), 4.20(\mathrm{~d}, J=$ $7.6 \mathrm{~Hz}, 1 \mathrm{H}), 4.62(\mathrm{~d}, J=11.2 \mathrm{~Hz}, 1 \mathrm{H}), 4.66(\mathrm{~d}, J=12.0 \mathrm{~Hz}, 1 \mathrm{H}), 4.80(\mathrm{~d}, J=12.0 \mathrm{~Hz}, 1 \mathrm{H}), 4.81(\mathrm{~d}$, $J=10.8 \mathrm{~Hz}, 1 \mathrm{H}), 4.91(\mathrm{~d}, J=11.2 \mathrm{~Hz}, 1 \mathrm{H}), 4.98(\mathrm{~d}, J=11.2 \mathrm{~Hz}, 1 \mathrm{H}), 4.99(\mathrm{~d}, J=3.2 \mathrm{~Hz}, 1 \mathrm{H})$, 7.27-7.37 (m, 15H); ${ }^{13} \mathrm{C} \mathrm{NMR}\left(100 \mathrm{MHz}, \mathrm{CDCl}_{3}\right) \delta$ 55.2, 58.2, 59.2, 60.4, 60.8, 66.1, 69.9, 70.1, 71.0, 73.3, 74.9, 75.8, 77.8, 79.4, 79.7, 81.5, 82.1, 83.0, 96.5, 97.8, 127.5, 127.5, 127.6, 127.8, 127.9, 128.0, 128.2, 128.2, 128.3, 137.9, 138.2, 138.5; IR (KBr) 2909, 1069, 740, $698 \mathrm{~cm}^{-1}$; LRMS (FAB) m/e $681\left(\mathrm{M}^{+}-\mathrm{H}\right), 543\left(\mathrm{M}^{+}-\mathrm{OMe}-\mathrm{BnOH}\right)$; HRMS (FAB) calcd for $\mathrm{C}_{38} \mathrm{H}_{49} \mathrm{O}_{11}\left(\mathrm{M}^{+}-\mathrm{H}\right)$ : 681.3275, found: 681.3275 .

Methyl-6- $O$-(2,3,4,6-tetra- $O$-methyl- $\beta$-d-glucopyranosyl)-2,3,4-tri- $O$-benzyl- $\alpha$-D-gluco pyranoside (21 $\beta){ }^{12}$ Prepared from $10(65.3 \mathrm{mg}, 0.199 \mathrm{mmol})$ and 19 (464.7 $\left.\mathrm{mg}, 1.000 \mathrm{mmol}\right)$ in the one-pot procedure, and purified with flash chromatography (hexane/EtOAc 5:3 to 1:1) and GPC (64.1 mg, 47\%, $\beta$ only). TLC $R_{f} 0.34$ (hexane/EtOAc 1:1); ${ }^{1} \mathrm{H}$ NMR (400 MHz, $\left.\mathrm{CDCl}_{3}\right) \delta 3.00-3.15$ (m, 3H), $3.24(\mathrm{ddd}, J=2.0,5.2,9.6 \mathrm{~Hz}, 1 \mathrm{H}), 3.36(\mathrm{~s}, 3 \mathrm{H}), 3.37(\mathrm{~s}, 3 \mathrm{H}), 3.48-3.59(\mathrm{~m}, 4 \mathrm{H}), 3.50$ (s, $3 \mathrm{H}), 3.56(\mathrm{~s}, 3 \mathrm{H}), 3.60$ (s, 3H), $3.66(\mathrm{dd}, J=4.4,10.8,1 \mathrm{H}), 3.77$, (ddd, $J=1.6,4.4,10.0,1 \mathrm{H}), 3.98$ $(\mathrm{dd}, J=9.2,9.6 \mathrm{~Hz}, 1 \mathrm{H}), 4.12(\mathrm{dd}, J=2.0,10.8 \mathrm{~Hz}, 1 \mathrm{H}), 4.20(\mathrm{~d}, 3.6 \mathrm{~Hz}, 1 \mathrm{H}), 4.59$ (d, $J=3.6 \mathrm{~Hz}$, $1 \mathrm{H}), 4.63(\mathrm{~d}, J=10.8 \mathrm{~Hz}, 1 \mathrm{H}), 4.64(\mathrm{~d}, J=12.4 \mathrm{~Hz}, 1 \mathrm{H}), 4.78(\mathrm{~d}, J=12.4 \mathrm{~Hz}, 1 \mathrm{H}), 4.81(\mathrm{~d}, J=$ $10.8 \mathrm{~Hz}, 1 \mathrm{H}), 4.88(\mathrm{~d}, J=10.8 \mathrm{~Hz}, 1 \mathrm{H}), 4.96(\mathrm{~d}, J=10.8 \mathrm{~Hz}, 1 \mathrm{H}), 7.26-7.35(\mathrm{~m}, 15 \mathrm{H}) ;{ }^{13} \mathrm{C} \mathrm{NMR}$ $\left(75 \mathrm{MHz}, \mathrm{CDCl}_{3}\right) \delta 55.1,59.4,60.4,60.7,60.8,68.3,69.8,71.5,73.4,74.8,74.9,75.8,78.0,79.6$, 79.8, 82.1, 83.5, 86.6, 98.0, 103.5, 127.6, 127.7, 127.8, 127.9, 128.1, 128.1, 128.4, 128.4, 138.1, 138.4, 138.7; IR (KBr) 2905, 1067, 739, $696 \mathrm{~cm}^{-1}$; LRMS (FAB) m/e $681\left(\mathrm{M}^{+}-\mathrm{H}\right), 543$ $\left(\mathrm{M}^{+}-\mathrm{OMe}-\mathrm{BnOH}\right)$; HRMS (FAB) calcd for $\mathrm{C}_{38} \mathrm{H}_{49} \mathrm{O}_{11}\left(\mathrm{M}^{+}-\mathrm{H}\right): 681.3275$, found: 681.3276 .

Phenylthio-6- $O$-(2,3,4,6-tetra- $O$-methyl- $\beta$-p-glucopyranosyl)-2,3,4-tri- $O$-benzyl- $\beta$ ucopyranoside (23). Prepared from $10(65.9 \mathrm{mg}, 0.201 \mathrm{mmol})$ and $22(540.2 \mathrm{mg} 0.995 \mathrm{mmol})$ in the one-pot procedure, and purified with flash chromatography (hexane/EtOAc $3: 1$ to 2:1) and GPC (116.4 mg, 76\%). This compound was characterized as a mixture of two diastereomers ( $\alpha: \beta=$ ca. 1:4 by ${ }^{1} \mathrm{H}$ NMR): spectrum data of the major diastereomer was presented below. TLC $R_{f} 0.18$ (hexane/EtOAc 3:1); ${ }^{1} \mathrm{H}$ NMR $\left(400 \mathrm{MHz}, \mathrm{CDCl}_{3}\right) \delta 3.02(\mathrm{dd}, J=8.0,8.0 \mathrm{~Hz}, 1 \mathrm{H}), 3.10-3.26(\mathrm{~m}$, $3 \mathrm{H}), 3.36(\mathrm{~s}, 3 \mathrm{H}), 3.42-3.90(\mathrm{~m}, 7 \mathrm{H}), 3.51(\mathrm{~s}, 3 \mathrm{H}), 3.51(\mathrm{~s}, 3 \mathrm{H}), 3.61(\mathrm{~s}, 3 \mathrm{H}), 4.17(\mathrm{~d}, J=10.8 \mathrm{~Hz}$, $1 \mathrm{H}), 4.24(\mathrm{~d}, J=7.6 \mathrm{~Hz}, 1 \mathrm{H}), 4.60-4.93(\mathrm{~m}, 7 \mathrm{H}), 7.20-7.55(\mathrm{~m}, 20 \mathrm{H}) ;{ }^{13} \mathrm{C} \mathrm{NMR}(100 \mathrm{MHz}$, $\left.\mathrm{CDCl}_{3}\right) \delta 59.3,60.3,60.5,60.7,68.4,71.2,74.5,74.8,75.3,75.8,77.9,78.6,79.3,80.7,83.5,86.2$, 86.6, 87.3, 103.3, 127.0, 127.5, 127.6, 127.6, 127.6, 127.9, 128.2, 128.2, 128.2, 128.6, 128.8, 131.3, 132.1, 133.7, 137.8, 138.0; IR (neat) 2900, 1063, 745, $694 \mathrm{~cm}^{-1}$; LRMS (FAB) m/e $761\left(\mathrm{MH}^{+}\right), 651$ $\left(\mathrm{M}^{+}-\mathrm{SPh}\right), 543\left(\mathrm{M}^{+}-\mathrm{SPh}-\mathrm{BnOH}\right)$; HRMS $(\mathrm{FAB})$ calcd for $\mathrm{C}_{43} \mathrm{H}_{53} \mathrm{O}_{10} \mathrm{~S}\left(\mathrm{MH}^{+}\right)$: 761.3359, found: 761.3355 .

${ }^{1} \mathrm{H}$ and ${ }^{13} \mathrm{C}$ NMR spectra of $\mathbf{2 1} \alpha, \mathbf{2 1} \beta$, and $\mathbf{2 3}$ (mixture of $\alpha$ and $\beta$ ) are as follows: 


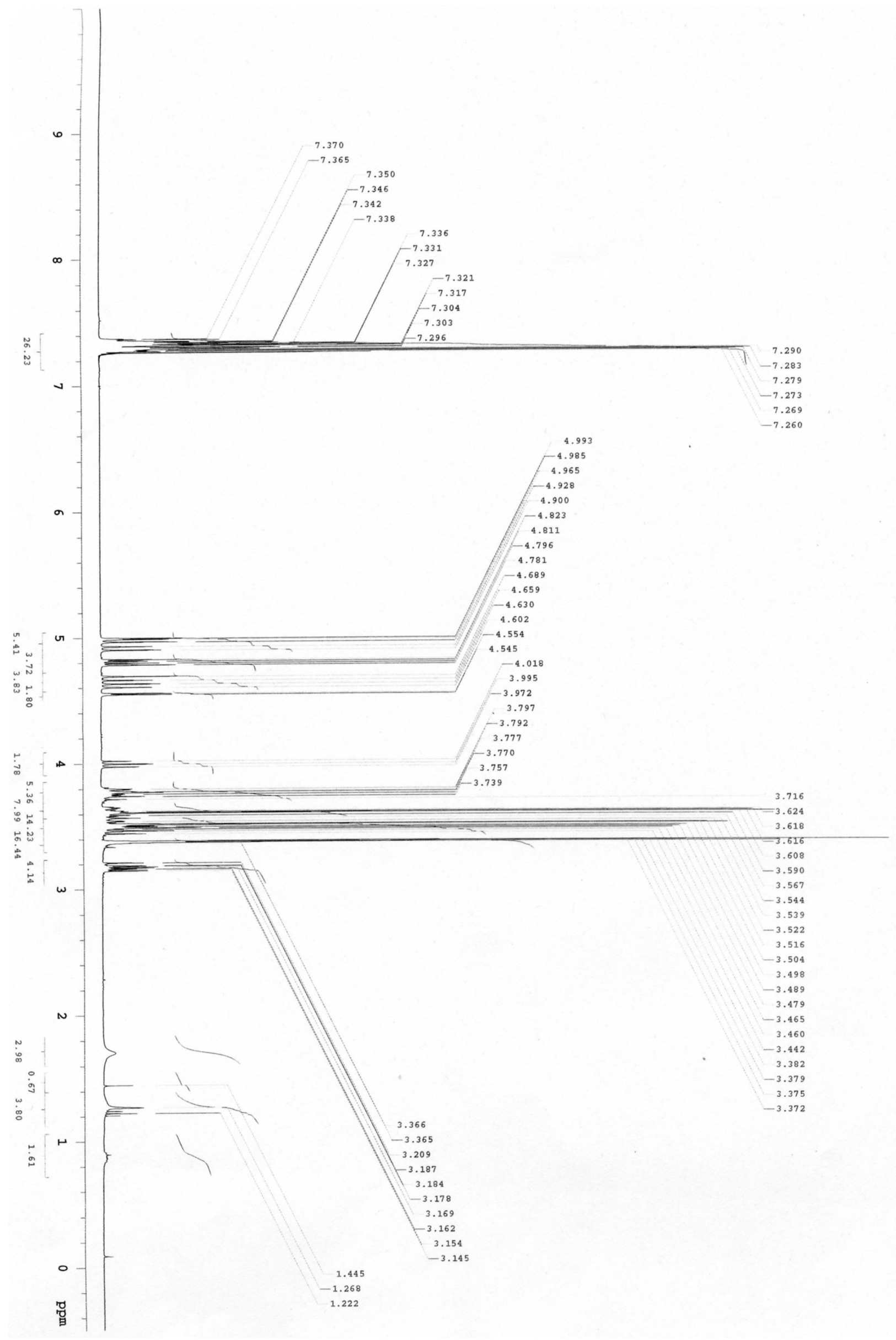

${ }^{1} \mathrm{H}$ NMR spectrum of $\mathbf{2 1} \alpha$. 


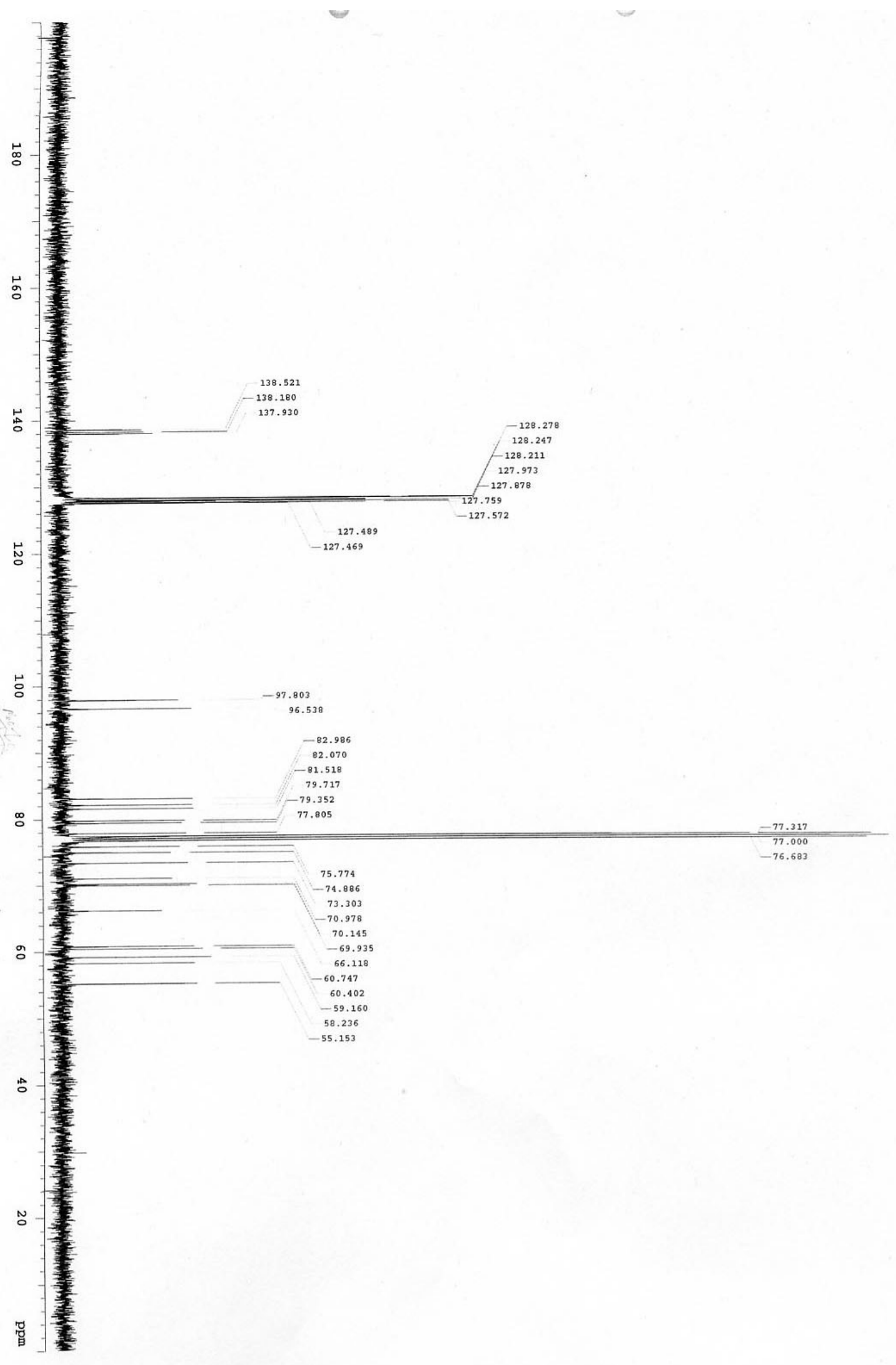

${ }^{13} \mathrm{C}$ NMR spectrum of $\mathbf{2 1} \alpha$. 


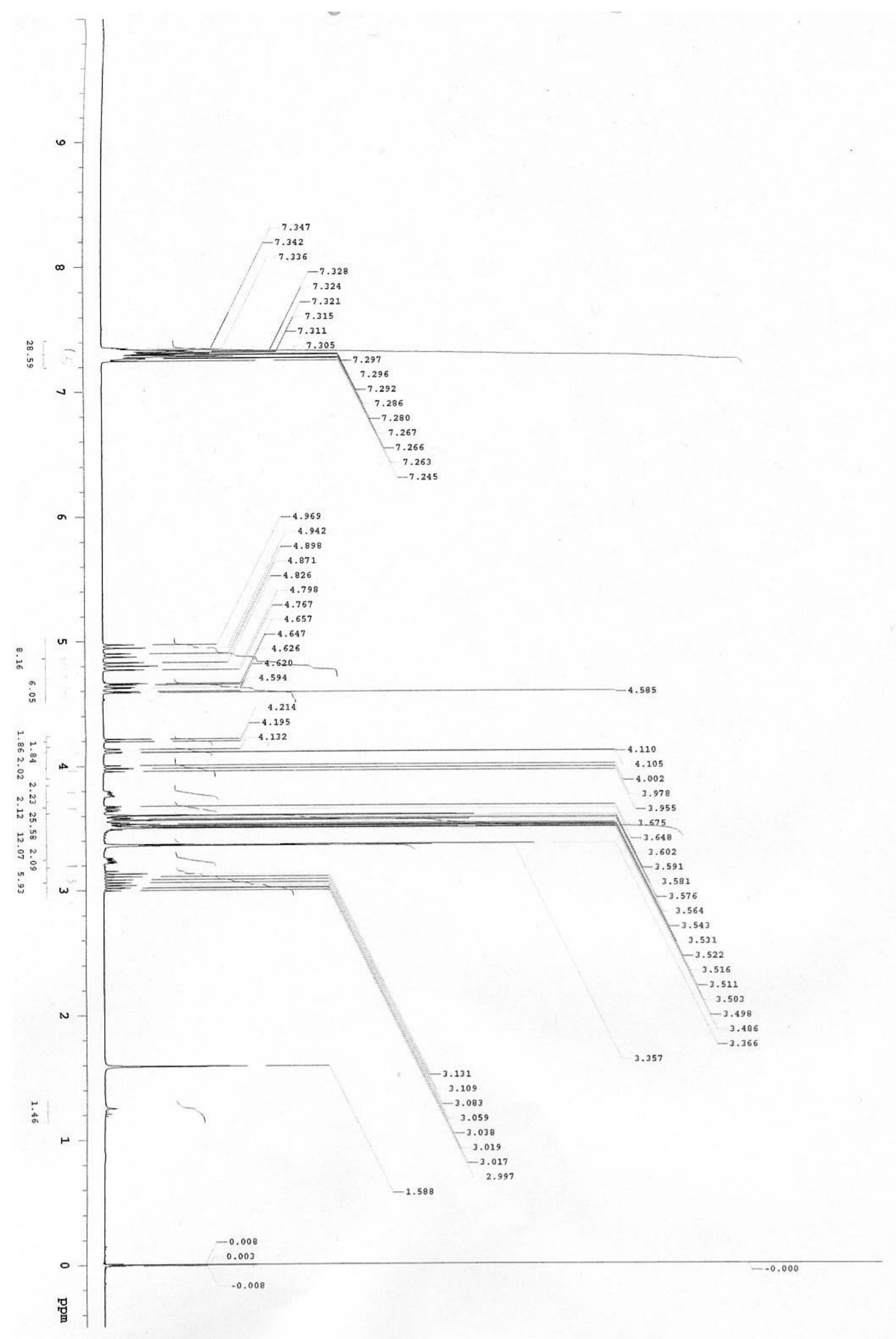

${ }^{1} \mathrm{H}$ NMR spectrum of $\mathbf{2 1} \beta$. 


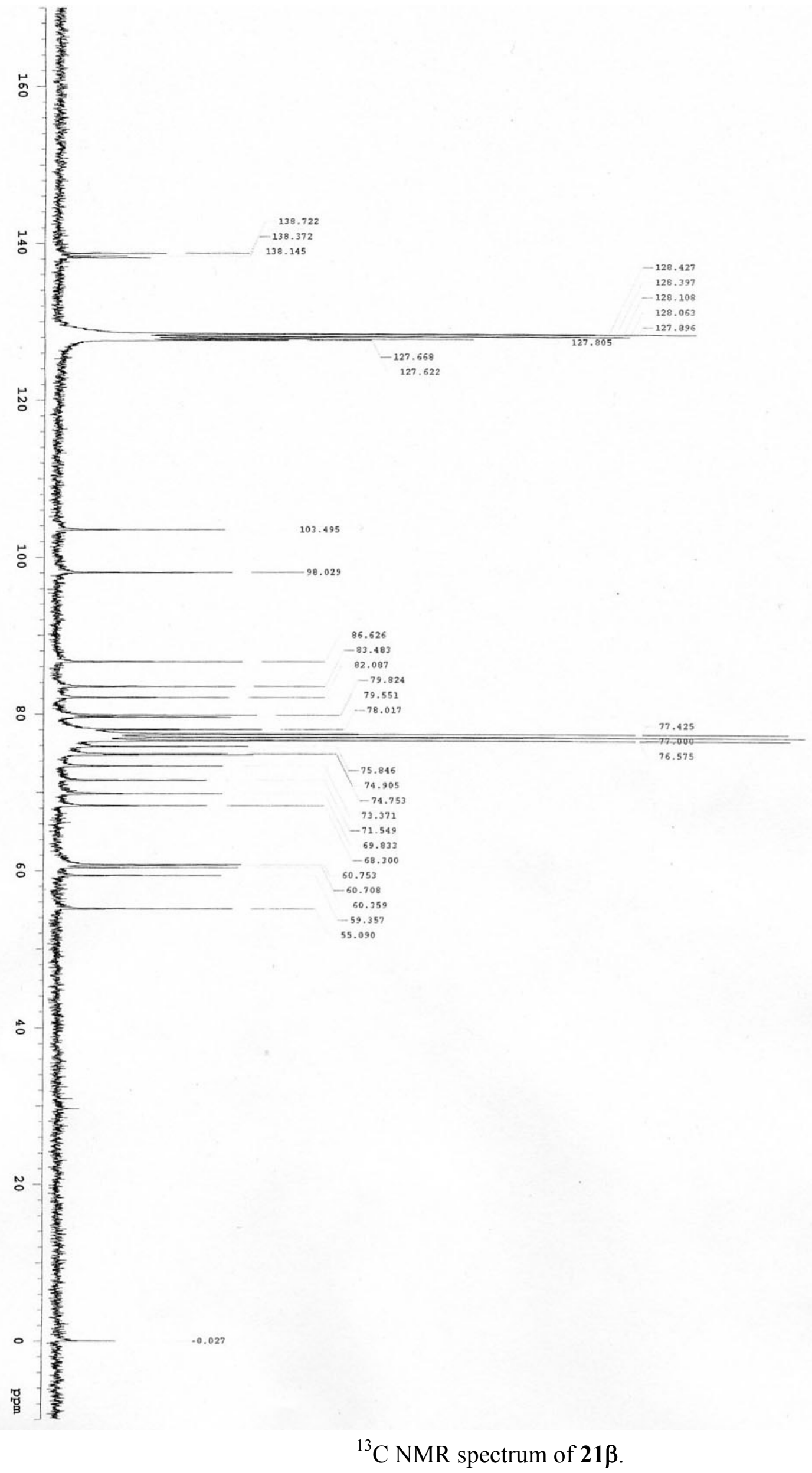




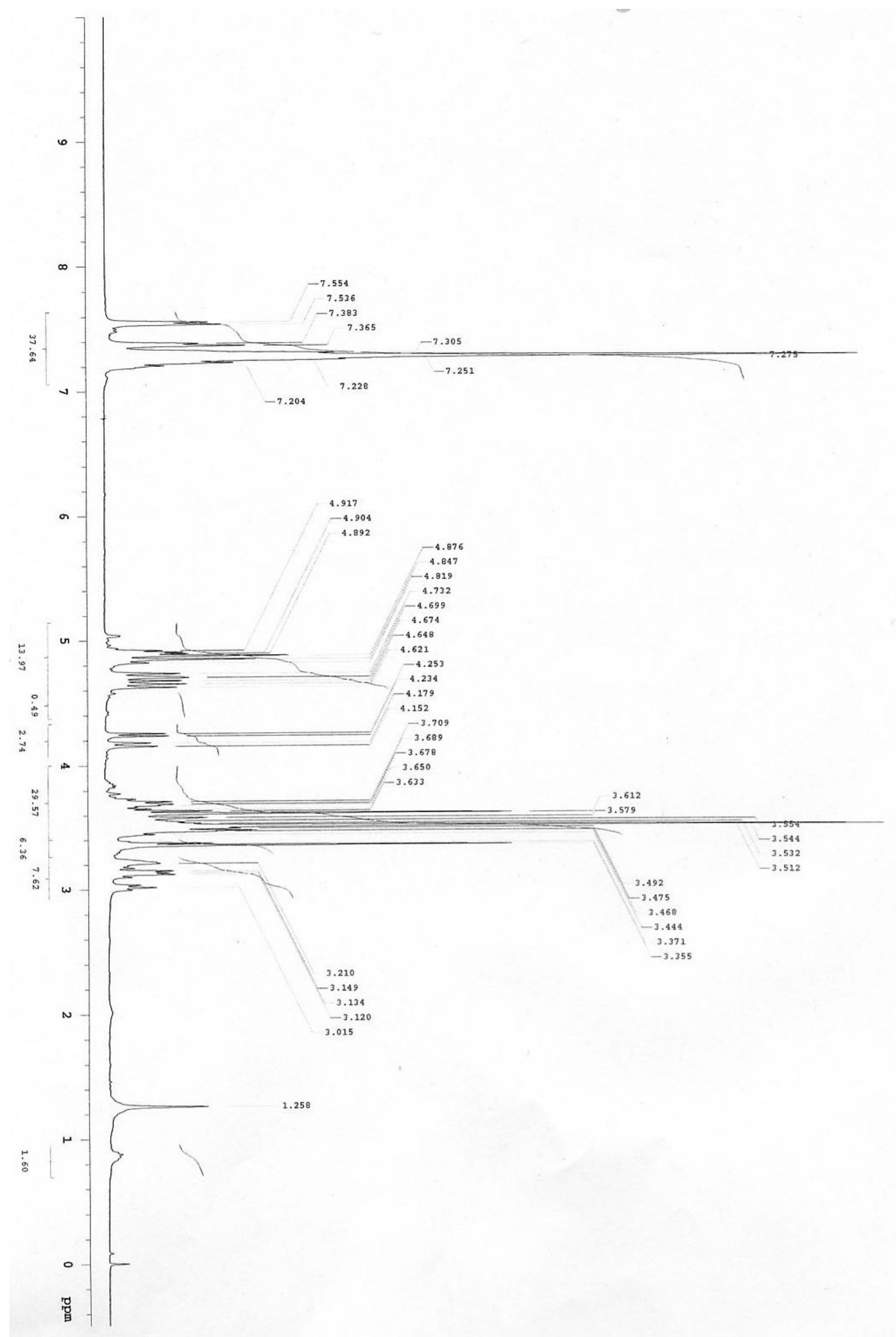

${ }^{1} \mathrm{H}$ NMR spectrum of $\mathbf{2 3}$. 


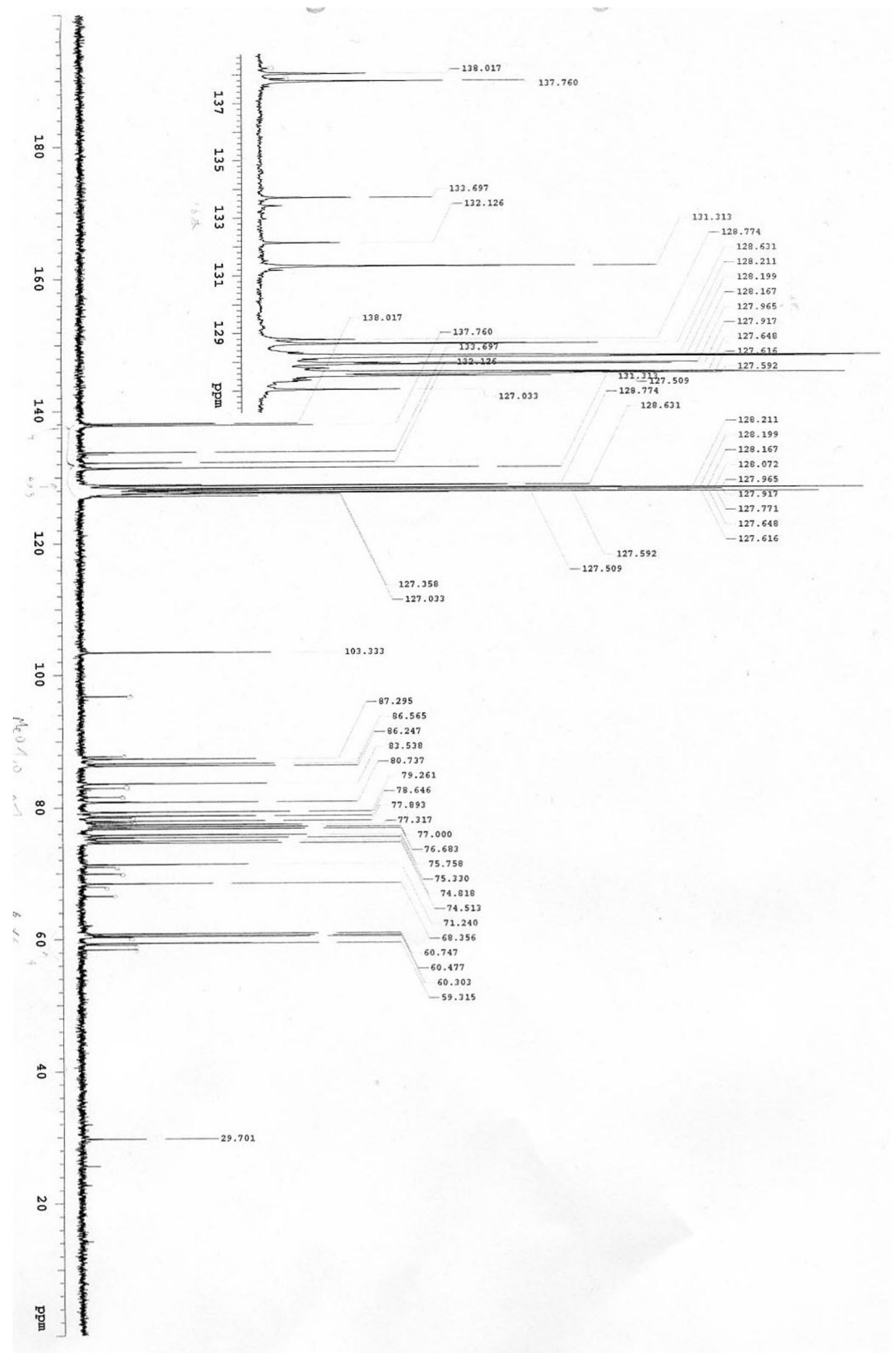

${ }^{13} \mathrm{C}$ NMR spectrum of $\mathbf{2 3}$. 


\section{References}

(1) Yoshida, J.; Sugawara, M.; Tatsumi, M.; Kise, N. J. Org. Chem. 1998, 63, 5950-5961.

(2) Braga, A. L.; Silveira, C. C.; Dornelles, L.; Zeni, G. ; Galarza, F. A. D.; Wessjohann, L. A. Syn. Commun. 1995, 25, 3155-3162.

(3) Mallet, J.-M.; Meyer, G.; Yvelin, F.; Jutand, A.; Amatore, C.; Sinay, P. Carbohydr. Res. 1993, 244, 237-246.

(4) Motawia, M. S.; Marcussen, J.; Møller B. L. J. Carbohydr. Chem. 1995, 14, 1279-1294.

(5) Miljković, M.; Habash-Marino, M. J. Org. Chem. 1983, 48, 855-860.

(6) Wipf, P.; Reeves, J. T. J. Org. Chem. 2001, 66, 7910-7914.

(7) Vankayalapati, H.; Singh, G.; Tranoy, I. Tetrahedron: Asymmetry 2001, 1373-1381.

(8) Kreuzer, M.; Thiem, J. Carbohydrate Res. 1986, 149, 347-361.

(9) Garcia, B. A.; Gin, D. Y. J. Am. Chem. Soc. 2000, 4269-4279.

(10) Thiem, J.; Wiesner, M. Synthesis 1988, 124-127.

(11) Garcia, B. A.; Poole, J. L.; Gin, D. Y. J. Am. Chem. Soc. 1997, 7597-7598.

(12) Selected data of ${ }^{1} \mathrm{H}$ and ${ }^{13} \mathrm{C}$ NMR was reported: Pan, S.; Li, H.; Hong, F.; Yu, B.; Zhao, K. Tetrahedron Lett. 1997, 38, 6139-6142.

(13) Suga, S.; Suzuki, S.; Yamamoto, A.; Yoshida, J. J. Am. Chem. Soc. 2000, 122, 10244-10245.

(14) Nicolaou, K. C.; Randall, J. L.; Furst, G. T. J. Am. Chem. Soc. 1985, 107, 5556-5558.

(15) Damager, I.; Olsen, C. E.; Møller, B. L.; Motawia, M. S. Carbohydrate Res. 1999, 320, 19-30.

(16) Li, P.; Sun, L.; Landry, D.; Zhao, K. Carbohydrate Res. 1995, 275, 179-184. 